\title{
Efectos de la reexperimentación emocional mediante lengua de señas colombiana sobre la sintomatología depresiva en personas sordas
}

\author{
Luis Miguel Moreno-Murcia ${ }^{\text {a }}$ Iván Felipe Medina-Arboleda ${ }^{\text {b* }}$
}

\author{
a Corporación Universitaria Minuto de Dios, Bogotá, Colombia

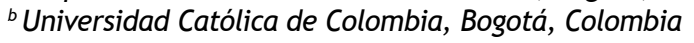

Recibido el 18 de marzo de 2020; aceptado el 30 de julio de 2020

\author{
PALABRAS CLAVE \\ Reexperimentación \\ emocional, depresión, \\ sordera, \\ estilo lingüístico, \\ LIWC
}

\begin{abstract}
Resumen Dada la prevalencia de la sintomatología depresiva en personas sordas y el déficit en técnicas de intervención, el propósito del estudio fue evaluar la efectividad de la reexperimentación emocional mediante lengua de señas colombiana en dos participantes sordas con sintomatología depresiva leve. Se utilizó un diseño experimental de caso único $-A B-$, con evaluaciones pre y posintervención de la sintomatología depresiva y seguimiento a las variaciones en el estilo lingüístico. La reexperimentación emocional disminuyó la sintomatología depresiva leve en las participantes, alcanzando niveles de no sintomatología al finalizar las sesiones y después de un mes de seguimiento. De acuerdo con el porcentaje de datos no solapados y el no solapamiento de todos los pares, el tratamiento tuvo un efecto medio. En términos de estilo lingüístico se discute el papel de las palabras de emociones negativas, emociones positivas, causalidad y el pronombre personal yo como promotoras de la recuperación. Finalmente, se sugieren investigaciones orientadas a la construcción de modelos de evaluación e intervención en comunidades con personas sordas.

(c) 2020 Fundación Universitaria Konrad Lorenz. Este es un artículo Open Access bajo la licencia CC BY-NC-ND (http://creativecommons.org/licenses/by-nc-nd/4.0/).
\end{abstract}

Effects of emotional disclosure through Colombian sign language on depressive symptoms in deaf people

Abstract Given the high prevalence of depressive symptoms in deaf people and the defi-
cit in intervention processes, the purpose of the study was to evaluate the effectiveness of
emotional disclosure using Colombian sign language in two deaf participants with mild de-
pressive symptoms. A single-case AB experimental design was used. Pre and post-interven-
tion variations in depressive symptoms were evaluated. Similarly, variations in linguistic
style were analyzed. The results indicate that emotional disclosure decreased mild depres-
sive symptoms in the two participants, reaching the rating of no symptoms at the end of

\section{KEYWORDS}

Emotional disclosure, depression, deaf, language style, LIWC

\footnotetext{
* Autor para correspondencia.

Correo electrónico: ifmedina@ucatolica.edu.co
} 
the sessions and after one month of follow-up. According to the percentage of non-overlapping data and non-overlapping of all pairs indices, the treatment had a medium effect. In terms of linguistic style, the role of negative emotion words, positive emotions, causality, and the personal pronoun I as promoters of recovery is discussed. New research aimed at building evaluation and intervention models in the deaf community is proposed.

(c) 2020 Fundación Universitaria Konrad Lorenz. This is an open access article under the CC BYNC-ND license (http://creativecommons.org/licenses/by-nc-nd/4.0/).

Los reportes clínicos sobre problemas de salud en pacientes que no expresan sus experiencias traumáticas llevaron a la formulación de la técnica de escritura emocional (apertura emocional o autorrevelación emocional), la cual consiste en la escritura de experiencias traumáticas, molestas o altamente estresantes, en sesiones de 20 minutos, por tres o cuatro días consecutivos (Pennebaker \& Beall, 1986). La expresión emocional en diferentes modalidades (oral, escrita, señada, etc.) induce al consultante a organizar en secuencias lógicas y coherentes los pensamientos y las emociones asociados a los eventos traumáticos, y así dar sentido a las experiencias (Pennebaker, 2011, 2018; Pennebaker \& Seagal, 1999).

Para analizar los efectos de las intervenciones se analizan, a su vez, las producciones textuales (o transcritas según la modalidad) con el programa Linguistic Inquiry and Word Count (LIWC). El programa cuenta las palabras en los textos y las compara con diccionarios preconfigurados (e.g., en la categoría Enojo hay más de 180 palabras) y computa la participación de frecuencias de la categoría en el total de palabras (Groom \& Pennebaker, 2002; Pennebaker, Mehl \& Niederhoffer, 2003).

La clasificación más amplia de las palabras es en palabras de función y contenido. Las palabras de contenido tienen un significado culturalmente compartido (e.g., sustantivos, verbos y adjetivos). Las palabras de función -0 estilo- organizan las producciones verbales (e.g., pronombres, artículos y preposiciones) (Pennebaker, 2011). Según Pennebaker et al. (2003), el uso de las palabras de función se asocia con la personalidad, la identidad social y el estado psicológico. En la versión en español, el programa abarca 72 categorías entre palabras de función y contenido, y en estas últimas constructos psicológicos de interés (Pennebaker et al., 2003; Ramírez-Esparza, Pennebaker, García \& Suriá, 2007).

Por medio del análisis de los textos, tras las intervenciones de experimentación y reexperimentación emocional, se han identificado algunas características asociadas a las mejoras en salud: (1) utilizar más palabras emocionales positivas (e.g., felicidad y optimismo); (2) usar un número moderado de palabras sobre afectividad negativa (e.g., tristeza y miedo); (3) aumentar progresivamente las palabras de la dimensión cognitiva, en particular de reflexión y autorreflexión (e.g., pensar y entender); (4) utilizar palabras causales que le dan coherencia a la narrativa (e.g., razón y causa); (5) alternar el uso de pronombres, en otras palabras, cambiar la conjugación mayoritaria en primera persona (yo, mí, nosotros, etc.) e incluir la segunda y tercera persona (ustedes, ella, ellos, etc.) (Fernández, Páez \& Pennebaker, 2004; Pennebaker, 2011; 2018).

\section{Reexperimentación emocional y depresión}

En población universitaria, Baum y Rude (2013) compararon la eficacia de la experimentación con escritura expresiva tradicional y la escritura expresiva en línea con instrucciones de aceptación de la emoción en la reducción de síntomas depresivos. Se encontró que las personas con bajos niveles de sintomatología depresiva se beneficiaron más de la escritura expresiva con instrucciones de aceptación de la emoción. Gortner, Rude y Pennebaker (2006), también con universitarios, reportaron la eficacia de la reexperimentación emocional tradicional en la disminución de síntomas depresivos, en particular en participantes con dificultades para expresar sus emociones y pensamientos.

En lengua castellana, Peñate, Del Pino y Bethencourt (2010) investigaron en universitarias el efecto de escribir sobre uno o diferentes traumas. Para esto tomaron medidas pre y posintervención de depresión, afecto positivo y negativo, entre otras. Las mediciones de depresión disminuyeron en todas las condiciones; en quienes se presentó la reducción habían escrito por más de 30 minutos. A su vez, Del Pino, Peñate, Fumero, Bethencourt y Zambrano (2016) investigaron los efectos de la escritura expresiva sobre depresión, afecto positivo y negativo, entre otras. Participaron 60 estudiantes universitarios asignados al azar a las condiciones escritura expresiva y de situaciones triviales. El grupo de escritura expresiva disminuyó de forma significativa sus niveles de depresión y afecto negativo, así como aumentó el afecto positivo.

En Corea del Sur, Lee et al. (2016) analizaron los efectos de la escritura expresiva en línea sobre los síntomas subsindrómicos depresivos. Los participantes (estudiantes universitarios) fueron asignados a las condiciones experimental (escritura expresiva) y control. La técnica contribuyó a la disminución significativa de sintomatología depresiva y se identificó que el uso de palabras emocionales y cognoscitivas se correlacionó con tal disminución.

Por su parte, Graf, Gaudiano y Geller (2008) reportaron los beneficios de la escritura expresiva sobre la sintomatología asociada a cuadros de depresión, ansiedad y estrés en consultantes de terapia ambulatoria. Los participantes fueron asignados a dos condiciones experimentales, grupos de escritura expresiva y control. El grupo de escritura expresiva mostró reducciones significativas en depresión $(F(1,41)=6.03, p<.05, \mathrm{n} 2=.13) ;$ ansiedad $(F(1,41)=4.93$, $p<.05, \mathrm{n} 2=.11)$ y estrés $(F(1,41)=7.16, p<.01, \mathrm{n} 2=.15)$ versus el control. A su vez, Di Blasio et al. (2015) documentaron la efectividad de la escritura expresiva en la disminución de síntomas depresivos durante el posparto. 
Smyth, Hockemeyer y Tulloch (2008) investigaron la eficacia de la intervención con escritura expresiva sobre el trastorno de estrés postraumático. Los participantes se asignaron aleatoriamente a los grupos experimental $n=15$ (escritura sobre evento traumático) y control $n=10$ (escritura temática neutral). Se tomaron medidas pre y posintervención (tres meses después). Pese a que no se presentaron reducciones significativas en sintomatología asociada al trastorno, el grupo experimental presentó reducciones significativas en depresión $(p<.10)$, tensión e ira $(p<.05)$.

Por su parte, Milbury et al. (2016) analizaron si los síntomas depresivos iniciales y el apoyo social moderan los efectos de la intervención de escritura expresiva en pacientes diagnosticados con cáncer de riñón. Los resultados indican que la interacción entre síntomas depresivos iniciales y apoyo social inicial fue significativa $(F(2.164)=21.16$, $p<.0001 ; r=.34)$. Los participantes del grupo de escritura expresiva presentaron síntomas depresivos más bajos durante el seguimiento cuando tenían alto apoyo social en comparación con el grupo control $(t=-2.72, p<.01)$.

Lorenz, Pulverman y Meston (2015) examinaron el vínculo de la escritura expresiva y la sintomatología depresiva en mujeres con antecedentes de contacto sexual no deseado. Los cambios en el estilo lingüístico asociados a la disminución de síntomas depresivos (comparación de escritos pre y pos), a saber, la menor utilización del pronombre yo $(F(1,969.02)$ $=4.36, p=.037, r=.33$ ) y el mayor uso de palabras de emoción positiva $(F(1,93.76)=4.56, p=.035, r=.37)$.

En contraste, se encuentran estudios que no han registrado efectos de las intervenciones basadas en reexperimentación emocional. Ireland, Malouff y Byrne (2007) no encontraron efectos sobre los indicadores de depresión; Sloan, Marx y Greenberg (2011) sobre la sintomatología de estrés postraumático y sintomatología depresiva; Bernard, Jackson y Jones (2006), así como Hevey y Wilczkiewicz (2014) sobre ansiedad y depresión.

En el metanálisis de los efectos de la escritura sobre los síntomas depresivos de Reinhold, Bürkner y Holling (2018) se reporta que la escritura expresiva no produce efectos significativos a largo plazo; no obstante, después de la última sesión de escritura se identificó un tamaño del efecto pequeño $(g=-.09,(95 \% \mathrm{Cl}[-.15,-.02], p=.006$ (una cola), $k=22, t<.01, r<.01)$ ). Los tamaños del efecto son significativamente mayores cuando se presentaron más sesiones $(b=-.03, z=-1.77, p=.038)$, la escritura se centró en un tema específico $(b=-.09, z=-2.05, p=.020)$, a mayor edad de los participantes $(b=-.005, z=-.24, p=.025) \mathrm{y}$ cuando la conformación de la muestra fue mayoritariamente femenina $(b=-.003, z=-2.91, p=.004)$. Por tanto, la reexperimentación emocional se puede considerar un tratamiento con evidencia moderada $(d=.16)$ para condiciones específicas (Pennebaker, 2018).

\section{Sordera y depresión}

La depresión, trastorno del estado de ánimo con afectaciones de relevancia subjetiva en la afectividad, genera disfuncionalidad en las áreas de ajuste de las personas (Abelló, Cortes, Fonseca, García \& Mariño, 2013), está asociada al suicidio y suele presentarse en comorbilidad con otros cuadros clínicos como la ansiedad (Toro, Avendaño-Prieto \& Vargas, 2020). Según la Encuesta Nacional de
Salud Mental (Gómez-Restrepo et al., 2015), los trastornos del estado del ánimo son los más prevalentes en la población adulta (6.7\%). La OMS (2017) reporta que hay más de 300 millones de personas con depresión en el mundo (4.4\%) y la incidencia aumentó en un 18.4 \% entre el 2005 y el 2015. El panorama se agudiza si las personas con sintomatología depresiva además tienen discapacidad auditiva.

En el mundo existen, aproximadamente, 466 millones de personas con pérdida de la audición (OMS, 2018); en Colombia, según el Insor (2016), se encuentran 455 718. En cuanto al diagnóstico, Estrada (2008) señala las dificultades en el reconocimiento de la afectación mental producto de las barreras de comunicación. En términos de etiología, según Casas, Linares, Lemos y Restrepo (2009), las personas con deficiencia auditiva presentan una mayor vulnerabilidad a desarrollar trastornos del estado del ánimo versus personas oyentes, probablemente por el aislamiento asociado a las barreras de comunicación (Dawes et al., 2015), razón por la cual la depresión es más prevalente en personas sordas que en oyentes (Adigun, 2017; Rostami, Bahmani, Bakhtyari \& Movallali, 2014) y se identifica a edades tempranas (Lier, 2013); sin embargo, la sintomalogía no difiere entre poblaciones (Bozzay et al., 2017; Cuenca, 2018; Masudul-haq, Shahid, Saqib \& Khalid, 2008).

Desafortunadamente, las intervenciones psicológicas adaptadas a personas sordas son escasas (Estrada, Beyebach, Delgado \& Freire, 2008), pese al mayor riesgo suicida de la población (Turner, Windfuhr \& Kapur, 2007). Bone (2019) entrevistó a seis participantes sordos (en Canadá) con diagnóstico de depresión mayor. Las narrativas convergen en las dificultades de entablar procesos terapéuticos y vincularse emocionalmente en estos por las barreras en la comunicación -con los terapeutas y con otros miembros de la comunidad de sordos- y el desconocimiento de los terapeutas de la lengua, la cultura y la comunidad sorda. Por tal razón, los participantes practican la "resiliencia por su cuenta” (e.g., a través del ejercicio físico).

En lengua castellana, Estrada et al. (2008) adaptaron el BDI-II a la lengua de señas de España -BDI-IIAS- e implementaron la terapia breve centrada en solución, la cual fue efectiva en tres pacientes con sintomatología depresiva. Esta intervención tomó entre cuatro y ocho sesiones, alrededor de cuatro meses y medio. Sin embargo, los autores destacan que, a pesar de la efectividad, la intervención fue mediada por intérpretes y no hubo un establecimiento de línea de base.

En el contexto de la evidencia disponible, la reexperimentación emocional se presenta como una alternativa de intervención de fácil aplicación, bajo costo y adaptabilidad lingüística para las personas sordas. En particular porque está documentado que las mayores ganancias de la intervención se dan en los participantes con dificultades para expresar sus emociones, pensamientos y sentimientos (Gortner et al., 2006; Peñate et al., 2010; Reinhold et al., 2018; Sedeño, 2011); a su vez, los estudios sobre aspectos que más afectan a la comunidad sorda en el acceso a salud señalan las barreras en la comunicación (Anderson, WolfCraig \& Ziedonis, 2017).

Así, entonces, hipotetizamos que una intervención que promueve la comunicación en la lengua de señas y que involucra contacto con personal capacitado en salud mental disminuirá la depresión leve, entre otras cosas, por la valoración positiva de la comunidad a las adaptaciones 
lingüísticas, en materiales y formatos, de las intervenciones en salud mental (Davidson, Cave, Reedman, Briffa \& Dark, 2012). En este contexto, el estudio evaluó la efectividad de la técnica de reexperimentación emocional, basada en el uso de la lengua de señas de Colombia, a fin de disminuir la sintomatología depresiva en personas sordas.

\section{Método}

\section{Tipo de investigación y diseño}

La investigación corresponde a un análisis intrasujeto con un diseño experimental de caso único $(A B)$. El estudio tuvo por propósito evaluar la efectividad de un tratamiento (Ato, López \& Benavente, 2013) bajo condiciones manipulativas y de control (Avendaño, Serrano, Toro \& Medina-Arboleda, 2018).

\section{Variables e instrumentos}

\section{Reexperimentación emocional}

Se aplicó el protocolo formulado por Pennebaker, Kiecolt-Glaser y Glaser (1988) bajo la perspectiva de la teoría de la inhibición de emociones, adaptado con base en los hallazgos sobre duración de sesiones, número de sesiones y tema de la reexperimentación (Peñate et al., 2010; Reinhold et al., 2018; Sedeño, 2011).

Las instrucciones del protocolo se adaptaron a lengua de señas colombiana y fueron señadas por una intérprete en cada sesión. La instrucción invitó a los participantes a expresar (en señas) sobre un hecho molesto o trauma, revelando la mayor cantidad de detalles sobre cómo se habían sentido y sobre las características de tiempo, lugar y protagonistas del hecho. A su vez, se especificó que la reexperimentación fuera abordada durante treinta minutos; no obstante, podían detenerse cuando lo consideraran conveniente. Las sesiones de reexperimentación tuvieron lugar durante cinco días consecutivos en una cámara de Gessel.

\section{Sintomatología depresiva}

La Escala de Depresión de Zung adaptada a lengua de señas colombiana es una escala de autorreporte tipo Likert de 20 preguntas. El instrumento original de Zung, en 1965, aborda la sintomatología depresiva sobre la base de aspectos afectivos, fisiológicos y psicológicos. La puntuación se obtiene de 1 a 4 para los ítems negativos y de 4 a 1 para los ítems positivos. La sumatoria de los puntajes determina el índice depresivo: en puntajes superiores al $50 \%$ se asume presencia de sintomatología depresiva (Lezama, 2012). La adaptación de la escala a lengua de señas colombiana cumple con los índices de confiabilidad (.63, alfa de Cronbach) y validez (.68, Spearman) (Quintero, Trujillo \& Reina, 2009).

\section{Estilo linguiístico}

Las sesiones de reexperimentación se registraron en video y un profesional de lengua de señas interpretó y transcribió a castellano el contenido señado. Las trascripciones se analizaron con el programa LIWC $®$ diccionario en español (Ramírez-Esparza et al., 2007).

\section{Participantes}

Los criterios de inclusión fueron: (1) diagnóstico de pérdida de la capacidad auditiva parcial (hipoacusia) o sordera total (cifosis); (2) puntuación en la Escala de Zung de 50- 59 (depresión leve); (3) no acudir actualmente a consulta por psicología; (4) saber leer y escribir en lengua castellana; (5) usar desde la infancia la lengua de señas. El criterio de inclusión de depresión leve se aplicó de acuerdo con las indicaciones de mayor ganancia terapéutica y menor riesgo iatrogénico para esta población. Finalmente, participaron dos estudiantes universitarias que cumplieron los criterios.

La participante 1, de 24 años, diagnosticada con sordera profunda desde el nacimiento por contagio de rubéola, a los cinco años aprendió la lengua de señas colombiana. Su estado civil es soltero y el nivel educativo es estudiante universitario. Convive con su madre y su abuelo. La participante 2 , de 25 años, fue diagnosticada con sordera profunda por fiebre en los primeros días de nacida. A los cinco años aprendió la lengua de señas colombiana. Su estado civil es unión libre y el nivel educativo es profesional. Convive con su pareja e hijo. Las participantes no asistían a terapia fonoaudiológica, médica u ocupacional.

\section{Aspectos éticos}

El proyecto de investigación fue aprobado por el Comité Institucional de Ética de la Universidad Católica de Colombia. Los participantes diligenciaron el consentimiento informado que incluyó el propósito de la investigación, la información de desistimiento, la identificación de los investigadores, la autorización de grabación en video y audio y las condiciones de seguimiento del proyecto.

\section{Procedimiento}

En primer lugar, se convocó a participar a la población sorda de una universidad colombiana en Bogotá. La socialización tuvo lugar en lengua de señas colombiana. Posteriormente, para evaluar los criterios de inclusión, los interesados respondieron la Escala de Zung (medición 1). Cuatro días después, las participantes que cumplieron los criterios de inclusión iniciaron la aplicación protocolo (previo diligenciamiento del consentimiento informado). Durante la intervención las participantes estuvieron acompañadas de un psicólogo y una psicóloga, a su vez profesional en lengua de señas, quien explicó el procedimiento, presentó las instrucciones del protocolo y aclaró las dudas e inquietudes de las participantes.

El equipo de investigación filmó las sesiones de evaluación e intervención. La psicóloga profesional en lengua de señas interpretó y transcribió la lengua de señas a castellano. Finalizada la primera sesión, las participantes respondieron la escala de depresión (medición 2). Las sesiones se aplicaron por cinco días consecutivos y al final del quinto día se aplicó la prueba de depresión (medición 3). Cuatro semanas después de la intervención los participantes cumplimentaron la última medición (4) de depresión.

\section{Resultados}

Para cada participante se presentan los registros de sintomatología depresiva, los análisis diferenciales entre sesiones, el tamaño del efecto y el análisis del estilo linguiístico. Al final se presenta la comparación intersujetos.

Las estimaciones del tamaño del efecto se calcularon con el porcentaje de datos no solapados (PND) y el no solapamiento de 
todos los pares (NAP) cuyas formulas son: $\mathrm{PND}=\mathrm{N}$ de datos del tratamiento que exceden el dato más extremo de la LB/total de datos del tratamiento x 100 (Scruggs \& Mastropieri, 1998) y $\mathrm{NAP}=(\mathrm{N}$. de pares sin solapamiento $)+(.5 \times \mathrm{N}$. de empates $) /$ total $N$. de pares de datos comparando la LB y el tratamiento $x$ 100 (Parker \& Vannest, 2009).

\section{Participante 1}

\section{Mediciones}

En la figura 1 se observan los puntajes de depresión. En la primera medición, la participante obtuvo 50/100 en la Escala de Zung (depresión leve). El puntaje aumentó tras la primera sesión (53/100; depresión leve). Al finalizar la intervención, la puntuación fue de 36/100 (sin sintomatología) y en el seguimiento fue de 45/100 (sin sintomatología). La duración de las sesiones fue en promedio de 29.2 minutos. El tiempo de reexperimentación disminuyó 35 minutos tras las sesiones 1 y 2; y tras las sesiones 3, 4 y 524,29 y 23 minutos, respectivamente. Si bien se presentó como instrucción que se señara durante 30 minutos, la participante finalizó las sesiones en los tiempos mencionados.

\section{Análisis de diferencias entre sesiones}

Dado que las mediciones en depresión no se distribuyeron de forma normal (Shapiro-Wilk con $p$ valor menor a .05), se usó la prueba no paramétrica de Friedman (equivalente a la prueba Anova para medidas repetidas). Se presentaron diferencias estadísticamente significativas entre todas las mediciones $($ chi cuadrado $=12.356 ; p=.006$ ).

\section{Tamaño del efecto}

El porcentaje de datos no solapados PND obtuvo valores en el rango de $50 \%-69 \%$, efectividad cuestionable (Scruggs \& Mastropieri, 1998). Por otra parte, el índice de no solapamiento de todos los pares NAP muestra que el efecto del tratamiento es de nivel medio (66\%-92\%) (Parker \& Vannest, 2009).

\section{Estilo linguiístico}

La narrativa de la participante tuvo como temas las relaciones familiares, muerte de personas cercanas, relaciones con pares, condición de sordera, problemas médicos, contexto laboral y los sueños. En la tabla 1 se presenta la variación porcentual de las categorías linguíísticas analizadas a través de las sesiones (variación porcentual de sesión versus la inmediatamente anterior) y al comparar la sesión de final con la inicial. Las principales variaciones positivas entre la primera y última sesión se presentaron en las categorías total de palabras (53.06 \%), muerte (400\%) y causa $(11.6 \%)$. Las variaciones negativas en las categorías triste $(-34.29 \%)$, emociones negativas (-20.39\%) y pronombres personales plural (-23.54\%).

\section{Participante 2}

\section{Mediciones}

En la primera medición, la puntuación fue de 56/100 en la Escala de Zung (sintomatología depresiva leve) (véase la figura 2). Esta clasificación se mantuvo en la primera sesión (55/100; depresión leve). Al finalizar la intervención, la puntuación fue de 43/100 (sin sintomatología), y en seguimiento 48/100 (Sin sintomatología). Las sesiones duraron en promedio 26 minutos. Hubo un aumento progresivo en las primeras cuatro sesiones $(22,25,27$ y 35 minutos, respectivamente) y una disminución en la última (21 minutos).

\section{Análisis de diferencias entre sesiones}

Se presentaron diferencias estadísticamente significativas entre todas las mediciones (prueba no paramétrica de Friedman, chi cuadrado $=14.487 ; p=.002$ ).

\section{Tamaño del efecto}

El porcentaje de datos no solapados PND (Scruggs \& Mastropieri, 1998) indica que el tratamiento es de efectividad cuestionable (valores entre 50 \%-69\%). El índice de no solapamiento de todos los pares NAP fue de efecto medio (66 \%-92 \%) (Parker \& Vannest, 2009).

\section{Estilo linguiístico}

La narrativa de la participante 2 tuvo como contenidos las relaciones familiares, cuadros de depresión e intento suicida, relaciones de pareja, relación con pares, condición

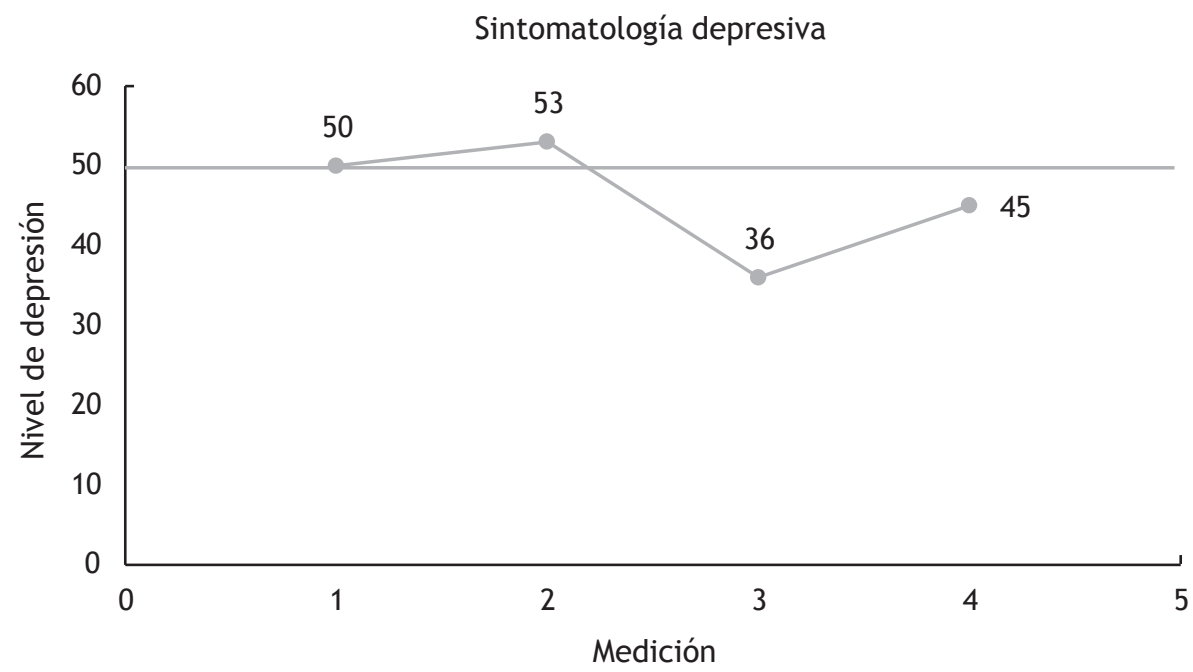

Figura 1. Sintomatología depresiva de la participante 1. 
Tabla 1 Variaciones porcentuales entre sesiones e inicio versus final en estilo lingüístico y contenido. Participante 1

\begin{tabular}{|c|c|c|c|c|c|c|}
\hline Categoría & $\mathrm{S} 1$ & $\mathrm{~S} 2$ & S3 & S4 & S5 & Final \\
\hline Cantidad de palabras & 2318 & $31.62 \%$ & $-23.57 \%$ & $65.65 \%$ & $-8.15 \%$ & $53.06 \%$ \\
\hline Pronombres personales singular & 14.37 & $14.27 \%$ & $-10.66 \%$ & $2.32 \%$ & $0.27 \%$ & $4.73 \%$ \\
\hline Yo & 9.45 & $14.07 \%$ & $-13.08 \%$ & $3.09 \%$ & $-0.83 \%$ & $1.38 \%$ \\
\hline Pronombres personales plural & 8.37 & $-15.77 \%$ & $-10.64 \%$ & $6.83 \%$ & $-4.90 \%$ & $-23.54 \%$ \\
\hline Afecto & 6.99 & $-7.58 \%$ & $1.55 \%$ & $-3.35 \%$ & $-9.31 \%$ & $-17.74 \%$ \\
\hline Emociones Positivas & 4.44 & $-4.05 \%$ & $14.79 \%$ & $-15.75 \%$ & $-8.25 \%$ & $-14.86 \%$ \\
\hline Emociones Negativas & 2.55 & $-13.73 \%$ & $-10.45 \%$ & $18.27 \%$ & $-12.88 \%$ & $-20.39 \%$ \\
\hline Enfado & 1.21 & $0.00 \%$ & $-14.88 \%$ & $18.45 \%$ & $-14.75 \%$ & $-14.05 \%$ \\
\hline Triste & 0.35 & $2.86 \%$ & $-52.78 \%$ & $23.53 \%$ & $9.52 \%$ & $-34.29 \%$ \\
\hline Metacognición & 28.26 & $7.86 \%$ & $-1.80 \%$ & $-4.08 \%$ & $-2.02 \%$ & $-0.46 \%$ \\
\hline Insight & 4.27 & $25.06 \%$ & $-20.41 \%$ & $18.12 \%$ & $-23.11 \%$ & $-9.60 \%$ \\
\hline Causa & 2.93 & $49.83 \%$ & $-4.33 \%$ & $0.48 \%$ & $-22.51 \%$ & $11.60 \%$ \\
\hline Muerte & 0.04 & $150.00 \%$ & $30.00 \%$ & $23.08 \%$ & $25.00 \%$ & $400.00 \%$ \\
\hline
\end{tabular}

de sordera y condiciones laborales. En la tabla 2 se presenta la variación porcentual de las categorías lingüísticas analizadas a través de las sesiones (variación porcentual de sesión versus la inmediatamente anterior) y al comparar la sesión de final con la inicial. Las principales variaciones positivas entre la primera y la última sesión se presentan en total de palabras (82 \%), emociones positivas (24\%) y pronombres personales en plural (53\%). Las categorías con mayor variación percentual negativa entre la primera y la última sesión fueron triste $(-47 \%)$, emociones negativas (-49\%), insight (-34\%) y yo $(-24 \%)$.

\section{Análisis intersujetos}

Las participantes disminuyeron los indicadores en depresión tras la intervención (Pre: $\mathrm{P} 1=50 ;$ P2 = 56; Pos:
$P 1=36 ; P 2=43)$. También en la prueba de seguimiento aumentaron sus puntaciones ( $\mathrm{P} 1=45-\mathrm{P} 2=48)$; sin embargo, se mantuvieron en la categoría no sintomatología. Las diferencias entre mediciones son estadísticamente significativas para depresión entre sesiones $(p<.05)$ y los análisis PND y NAP indican que la intervención tuvo un efecto medio y efectividad cuestionable.

Con respecto al estilo lingüístico, las comunalidades de variación positiva se presentan en las categorías cantidad de palabras y las negativas en afecto, emociones negativas, enfado, triste e insight. En cuanto a las discrepancias, están en pronombres personales plural (aumentan en la participante 2 y disminuyen en la participante 1), emociones positivas (aumentan en la participante 2 y disminuyen en la participante 1) y muerte (aumenta en la participante 1 y disminuye en la participante 2 ).

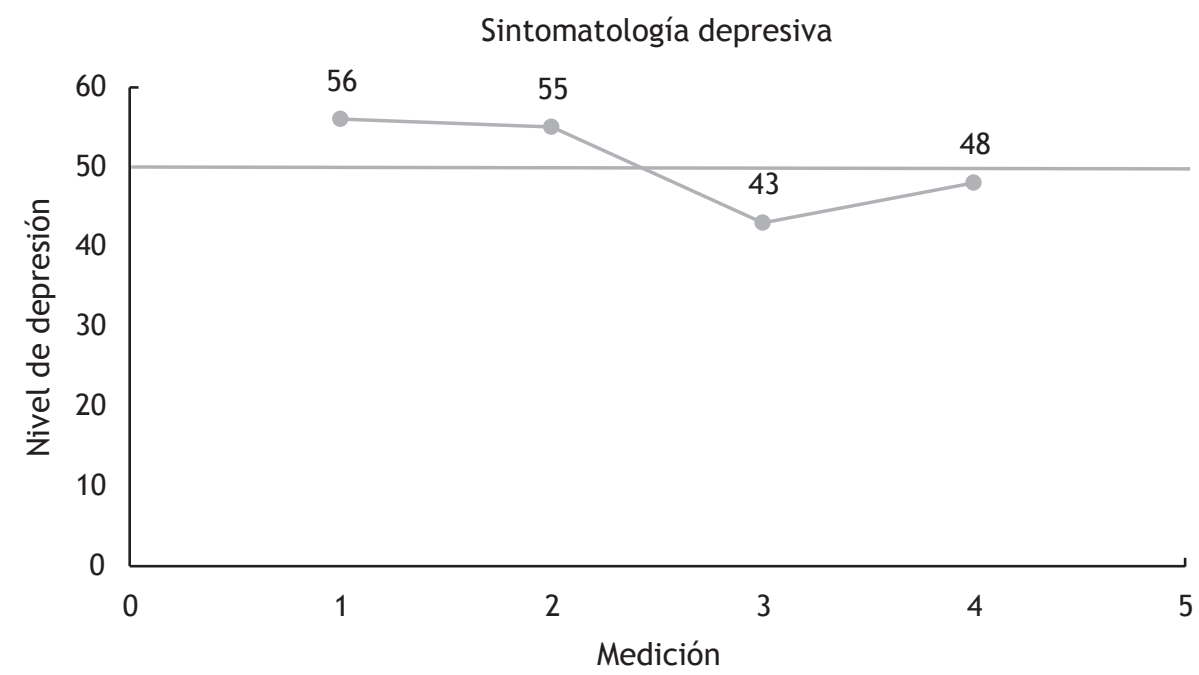

Figura 2. Sintomatología depresiva de la participante 2. 
Tabla 2 Variaciones porcentuales entre sesiones e inicio versus final en estilo lingüístico y contenido. Participante 2

\begin{tabular}{|c|c|c|c|c|c|c|}
\hline Categoría & $\mathrm{S} 1$ & $\mathrm{~S} 2$ & S3 & S4 & S5 & Final \\
\hline Cantidad de palabras & 1050 & $111.14 \%$ & $19.12 \%$ & $19.54 \%$ & $-39.59 \%$ & $81.62 \%$ \\
\hline Pronombres personales singular & 17.05 & $-11.61 \%$ & $4.25 \%$ & $11.27 \%$ & $-13.62 \%$ & $-11.44 \%$ \\
\hline Yo & 12 & $-22.58 \%$ & $7.21 \%$ & $2.41 \%$ & $-10.59 \%$ & $-24.00 \%$ \\
\hline Pronombres personales plural & 5.14 & $25.49 \%$ & $9.15 \%$ & $4.83 \%$ & $6.64 \%$ & $53.11 \%$ \\
\hline Afecto & 7.24 & $-28.31 \%$ & $7.90 \%$ & $-4.46 \%$ & $5.79 \%$ & $-21.82 \%$ \\
\hline Emociones Positivas & 2.67 & $6.37 \%$ & $1.41 \%$ & $-13.19 \%$ & $32.00 \%$ & $23.60 \%$ \\
\hline Emociones Negativas & 4.57 & $-42.67 \%$ & $4.20 \%$ & $11.36 \%$ & $-13.82 \%$ & $-42.67 \%$ \\
\hline Enfado & 1.52 & $-1.97 \%$ & $-13.42 \%$ & $27.91 \%$ & $-30.30 \%$ & $-24.34 \%$ \\
\hline Triste & 0.19 & $42.11 \%$ & $-29.63 \%$ & $-15.79 \%$ & $-37.50 \%$ & $-47.37 \%$ \\
\hline Metacognición & 29.81 & $-2.72 \%$ & $7.97 \%$ & $-7.95 \%$ & $1.35 \%$ & $-2.01 \%$ \\
\hline Insigth & 4.95 & $-18.99 \%$ & $-6.48 \%$ & $-21.33 \%$ & $10.17 \%$ & $-34.34 \%$ \\
\hline Causa & 3.9 & $5.13 \%$ & $11.71 \%$ & $-4.59 \%$ & $-14.87 \%$ & $-4.62 \%$ \\
\hline Muerte & 0 & & $120.00 \%$ & $-9.09 \%$ & $-50.00 \%$ & \\
\hline
\end{tabular}

\section{Discusión}

El estudio evaluó los efectos de la reexperimentación emocional, mediante lengua de señas, sobre la reducción de sintomatología depresiva en dos participantes diagnosticadas con hipoacusia. Se encontró que la intervención disminuyó la sintomatología depresiva: las mediciones pre y pos, los análisis intrasujeto e intersujetos, los índices del tamaño del efecto así lo sugieren; sin embargo, la efectividad es media o cuestionable. Hasta donde nos es conocido, este es el primer estudio en el que se documenta la efectividad de la reexperimentación con esta modalidad lingüística.

De acuerdo con Pennebaker y Beall (1986), la reexperimentación emocional promueve que el individuo organice, razone, exprese y comprenda pensamientos, emociones y sentimientos relacionados con experiencias traumáticas. Estos efectos se fundamentan, entre otros, en la toma de perspectiva (disminución de las palabras con pronombre yo) (Tackman et al., 2019), la revelación emocional inicial (aumento del malestar psicológico en las primeras sesiones para posteriormente una disminución en la frecuencia de palabras negativas) (Del Pino et al., 2016 ), la coherencia en el relato (aumento de las palabras de causalidad y epifanía) y la revaloración de eventos (aumento paulatino de palabras de emociones positivas) (Pennebaker, 2011).

Los valores de las diferencias en los puntajes de la sintomatología depresiva son consistentes con Reinhold et al. (2018), a saber, cambios significativos después de la última sesión de intervención. Los trabajos coinciden en el tema específico de la narrativa, género femenino de las participantes y el número de sesiones. La reducción de la sintomatología depresiva también es coherente con los resultados de Graf et al. (2008) y los de Smyth, Hockemeyer y Tulloch (2008). A su vez, el estudio replica los hallazgos de la técnica en depresión con jóvenes universitarios (Baum \& Rude, 2013; Del Pino, et al., 2016; Gortner et al., 2006; Lee et al., 2016; Peñate et al., 2010).
En relación con la narrativa del hecho traumático (un tema), los hallazgos concuerdan con la evidencia disponible sobre personas con puntaciones de depresión leve (Baum \& Rude, 2013; Del Pino et al., 2016; Di Blasio et al., 2015; Krpan et al.,2013; Peñate et al., 2010; Smyth et al., 2008). Por otra parte, una de las hipótesis de la efectividad de la reexperimentación considera que los más beneficiados son participantes con dificultades de expresión emocional o de comunicación (Gortner et al., 2006; Pennebaker, 2011), por tanto, la investigación aporta evidencia a favor del efecto positivo de la comunicación, dadas las barreras lingüísticas de la población.

En cuanto a la durabilidad de los efectos, Krpan et al. (2013) afirmaron que la disminución de los índices en depresión persistió por cuatro semanas, como en este caso. Sin embargo, los puntajes en depresión en el estudio aumentaron en el seguimiento, aunque no alcanzaron el rango de depresión leve. Por esta razón es importante evaluar con una mayor cantidad de seguimientos la durabilidad del efecto.

En cuanto al análisis del estilo lingüístico, las participantes disminuyeron la utilización de palabras en categorías como emociones negativas, enfado y tristeza, y aumentaron la frecuencia en la utilización de emociones positivas en la participante 2 y causa en la participante 1 . La variación de las frecuencias en emociones negativas es característica en las intervenciones de la sintomatología depresiva (Beléndez \& Suría, 2011). Ahora bien, no se identificaron los cambios en el uso del pronombre personal yo y emociones positivas (participante 1) y el aumento en causalidad (participante 2). Sin embargo, dados los efectos específicos de aumento y la posterior disminución en ambos casos de las puntuaciones en depresión, se hipotetiza el papel central del habla emocional, es decir, las variaciones en esta categoría independientemente de si aumentan o disminuyen la frecuencia (Lee et al., 2016). 
En futuras investigaciones es importante profundizar en las variaciones en las categorías de pronombre personal yo, causalidad y emociones positivas, a fin de dilucidar las vías de modulación de la respuesta emocional y sus correlatos en el estilo lingüístico. A su vez, el vínculo de la modulación emocional con otras áreas de ajuste de los participantes, por ejemplo, la actividad física (Bone, 2019; Gómez-Acosta \& Londoño-Pérez, 2020).

Por otra parte, la frecuencia del pronombre yo disminuyó en la participante 2 , lo cual se relaciona con lo registrado por Pulverman, Lorenz y Meston (2015), quienes encontraron una relación estadísticamente significativa entre la disminución de sintomatología depresiva y la disminución del uso del pronombre $(F(1,969.02)=4.36, p=.037$, $r=.33$ ). Tackman et al. (2019) encontraron que la expresión en primera persona $(r=.10,95 \% \mathrm{Cl}[.07, .13])$ y la alta frecuencia de emociones negativas son los marcadores de la propensión a la angustia; por tanto, al disminuir su frecuencia es coherente la disminución en depresión y ansiedad (Toro et al., 2020). También se encontró en la participante 2 el aumento de palabras positivas al igual en el estudio de Pulverman et al. (2015) $(F(1,93.76)=4.56, p=.035, r=.37)$.

Un resultado particular es el aumento en la categoría muerte (participante 2). Si bien la subdimensión muerte es más frecuente en relatos de personas con sintomatología depresiva en comparación con otros temas (Beléndez \& Suría, 2011) y se asocia con intentos suicidas (Fernández, 2015), para el caso el aumento obedece al contenido de asuntos personales (Pennebaker et al., 2003; Ramírez-Sparza et al., 2007), pues se alude a la muerte de un familiar, de lo cual más que una situación de alerta suicida se asocia con la tristeza por la pérdida de un ser querido.

En cuanto a las limitaciones, la primera es el tamaño de la muestra. Las dificultades de acceso a la población objetivo restringieron el número de participantes. Con el propósito de aumentar la replicabilidad y el estudio de factores asociados con la efectividad es fundamental replicar el protocolo. Otra limitación es la confiabilidad (aceptable) del instrumento de evaluación de la depresión que se usó, con las precauciones sobre la medida que conlleva.

En síntesis, la reexperimentación emocional con lengua de señas colombiana en cuadros depresivos leves se presenta como una alternativa de intervención con evidencia clínicamente relevante que permite garantizar una mayor equidad, en atención a la salud mental de una población históricamente marginada del sistema público y privado en salud, lo cual hace el sistema más justo (Sarmiento \& Yáñez, 2019).

\section{Referencias}

Abello, D., Cortés, O., Fonseca, L., García, P., \& Mariño, J. (2013). Escala multidimensional de trastornos afectivos-EMTA: análisis desde la teoría clásica de los test y la teoría de respuesta al ítem. Suma Psicológica, 20(2), 203-216. https://doi. org/10.14349/sumapsi2013.1442

Adigun, O. T. (2017) Depression and individuals with hearing loss: a systematic review. Journal of Psychology \& Psychotherapy, 7(5), 323. https://doi.org/10.4172/2161-0487.1000323

Anderson, M. L., Wolf-Craig, K. S., \& Ziedonis, D. M. (2017). Deaf people's help-seeking following trauma: experiences with and recommendations for the Massachusetts behavioral health care system. Psychological Trauma: Theory, Research, Practice, and Policy, 9(2), 239-248. https://doi.org/10.1037/tra0000219
Ato, M., López, J., \& Benavente, A. (2013). Un sistema de clasificación de los diseños de investigación en psicología. Anales de Psicología, 29(3), 1038-1059. https://doi.org/10.6018/analesps.29.3.178511

Avendaño, B. L., Serrano, S., Toro, R., \& Medina-Arboleda, I. F. (2018) Una perspectiva dimensional de las estrategias de análisis utilizadas en la investigación en psicología. En V. Sánchez, M. I. Barreto, \& B. Avendaño (Eds.), Investigación en psicología: aplicaciones e intervenciones (pp. 23-32). Bogotá: Universidad Católica de Colombia.

Baum, E. S., \& Rude, S. S. (2013). Acceptance-enhanced expressive writing prevents symptoms in participants with low initial depression. Cognitive Therapy and Research, 37(1), 35-42. https:// doi.org/10.1007/s10608-012-9435-x

Beléndez, M., \& Suriá, R. (2011). Los foros de internet para trastornos de ansiedad y depresión: un análisis comparativo del uso del lenguaje. Ansiedad y Estrés, 17, 15-25.

Bernard, M., Jackson, C., \& Jones, C. (2006). Written emotional disclosure following first-episode psychosis: effects on symptoms of post-traumatic stress disorder. British Journal of Clinical Psychology, 45, 403-415. https://doi.org/10.1348/014466505X68933

Bone, T. (2019). No one is listening: members of the deaf community share their depression narratives. Social Work in Mental Health, 17(1), 1-22. https://doi.org/10.1080/15332985.2018.149 8045

Bozzay, M. L., O’Leary., N., De Nadai, A. S., Gryglewicz, K., Romero, G., \& Karver, M. S. (2017). Adolescent depression: differential symptom presentations in deaf and hard-of-hearing youth using the Patient Health Questionnaire-9. Journal of Deaf Studies and Deaf Education, 22(2), 195-203. https://doi. org/10.1093/deafed/enw099

Casas, D., Linares, M., Lemos, \& Restrepo, D. (2009). Depresión y ansiedad en personas con deficiencia auditiva: revisión de literatura. Revista Virtual Universidad Católica del Norte, 28, 1-15.

Cuenca, A. (2018). Estudio correlacional de niveles de depresión y ansiedad en adultos con hipoacusia (Tesis de pregrado). Universidad del Azuay, Cuenca, Ecuador.

Davidson, F., Cave, M., Reedman, R., Briffa, D., \& Dark, F. (2012). Dialectical behavioral therapy informed treatment with deaf mental health consumers: an Australian pilot program. Australasian Psychiatry, 20(5), 425-428. https://doi. org/10.1177/1039856212458981

Dawes, P., Emsley, R., Cruickshanks, K., Moore, D., Fortnum, H., Edmondson-Jones, M.,...\& Munro, K. (2015). Hearing loss and cognition: the role of hearing aids, social isolation and depression. PLOS ONE, 10(3), 1-9. https://doi.org/10.1371/journal. pone.0119616

Del Pino, T., Peñate, W., Fumero, A., Bethencourt, J. M., \& Zambrano, S. (2016). La eficacia de la reexperimentación emocional el papel del optimismo y la alexitimia, Journal of Educational Psychology, 6(3), 193-205. https://doi.org/10.30552/ejihpe.v6i3.179

Di Blasio, P., Camisasca, E., Caravita, S. C. S., Ionio, C., Milani, L., \& Valtolina, G. G. (2015). The effects of expressive writing on postpartum depression and posttraumatic stress symptoms. Psychological Reports, 117(3), 856-882. https://doi. org/10.2466/02.13.PR0.117c29z3

Estrada, B. (2008) Salud mental: depresión y sordera. Monterrey, México: Universidad Autónoma de Nueva León.

Estrada, B., Beyebach, M., Delgado, C., \& Freire, R. (2008). Evaluación y tratamiento de la sintomatología depresiva en personas sordas. Psicología y Salud, 18, 99-106.

Fernández, M. (2015). Suicidio y escritura: un análisis clínico-lingüístico de textos y notas suicidas (Tesis doctoral). Universidad de Santiago de Compostela, España.

Fernández, I., Páez, D., \& Pennebaker, J. (2004). Escritura expresiva, deber de memoria y afrontamiento tras el impacto del 11-M: un estudio experimental. Ansiedad y Estrés, 10, 233-245. 
Gómez-Acosta, A., \& Londoño-Pérez, C. (2020). Emotion regulation and healthy behaviors of the body energy balance in adults: a review of evidence. Acta Colombiana de Psicología, 23(1), 366382. https://doi.org/10.14718/ACP.2020.23.2.14

Gómez-Restrepo, C., De Santacruz, C., Rodríguez, M. N., Rodríguez, V., Tamayo-Martínez, N.,...\& González, L. M. (2016). Encuesta Nacional de Salud Mental Colombia 2015. Protocolo del estudio. Revista Colombiana de Psiquiatría, 45, 2-8. https://doi. org/10.1016/j.rcp.2016.04.007

Gortner, E. M., Rude, S. S., \& Pennebaker, J. W. (2006). Benefits of expressive writing in lowering rumination and depressive symptoms. Behavior Therapy, 37(3), 292-303. https://doi.org/10.1016/j. beth.2006.01.004

Graf, M. C., Gaudiano, B. A., \& Geller, P. A. (2008). Written emotional disclosure: a controlled study of benefits of expressive writing homework in outpatient psychotherapy. Psychotherapy Research, 18, 389-399. https://doi. org/10.1080/10503300701691664

Groom, C. J., \& Pennebaker, J. W. (2002). Words. Journal of Research in Personality, 36, 615-621. https://doi.org/10.1016/ S0092-6566(02)00512-3

Hevey, D., \& Wilczkiewicz, E. (2014). Changes in language use mediate expressive writing's benefits on health-related quality of life following myocardial infartion. Health Psychology and Behavioral Medicine, 2(1), 1053-1066. https://doi.org/10.1080/216 42850.2014.971801

Ireland, M., Malouff, J., \& Byrne, B. (2007). The efficacy of written emotional expression in the reduction of psychological distress among police officers. International Journal of Police Science \& Management, 9(4), 303-311. https://doi.org/10.1350/ ijps.2007.9.4.303

Insor (Instituto Nacional para Sordos). (2016). Estadísticas básicas de la población sorda colombiana. Recuperado de http://www. insor.gov.co/observatorio/estadisticas-basicas-poblacion-sorda-colombiana/

Krpan, K. M., Kross, E., Berman, M. G., Deldin, P. J., Askren, M. K., \& Jonides, J. (2013). An everyday activity as a treatment for depression: the benefits of expressive writing for people diagnosed with major depressive disorder. Journal of Affective Disorders, 150(3), 1148-1151. https://doi.org/10.1016/j. jad.2013.05.065

Lee, S. W., Kim, I., Yoo, J., Park, S., Jeong, B., \& Cha, M. (2016). Insights from an expressive writing intervention on Facebook to help alleviate depressive symptoms. Computers in Human Behavior, 62, 613-619. https://doi.org/10.1016/j.chb.2016.04.034

Lezama, R. (2012). Propiedades psicométricas de la escala de Zung para síntomas depresivos en población adolescente escolarizada colombiana. Psychologia. Avances de la disciplina, 6(1), 91-101.

Lier, F. B. (2013). Mental health in deaf and hearing impaired children and adolescents (Tesis de maestría). Universidad de Oslo, Oslo, Noruega.

Masud-Ul-Haq, M., Shahid, H., Saqib, K., \& Khalid, M. (2008). Deafness and depression. Biomédica, 24,143-146.

Milbury, K., Lopez, G., Spelman, A., Wood, C., Matin, S., Tannir, N., ..., \& Cohen, L. (2016). Examination of moderators of expressive writing in patients with renal cell carcinoma: the role of depression and social support. Psychooncology, 26, 1361-1368. https://doi.org/10.1002/pon.4148

OMS (Organización Mundial de la Salud). (2017). Depression and other common mental disorders global health estimates. Recuperado de http://apps.who.int/iris/handle/10665/254610

OMS (Organización Mundial de la Salud). (2018). Sordera y pérdida de la audición. Recuperado de http://www.who.int/es/newsroom/fact-sheets/detail/deafness-and-hearing-loss
Parker, R. I., \& Vannest, K. J. (2009). An improved effect size for single case research: nonoverlap of all pairs (NAP). Behavior Therapy, 40, 357-367. https://doi.org/10.1016/j.beth.2008.10.006

Pennebaker, J. W. (2011). The secret life of pronouns. Nueva York: Bloomsbury Press.

Pennebaker, J. W. (2018). Expressive writing in psychological science. Perspectives on Psychological Science, 13(2), 226-229. https://doi.org/10.1177/1745691617707315

Pennebaker, J. W., \& Beall, S. K. (1986). Confronting a traumatic event: toward an understanding of inhibition and disease. Journal of Abnormal Psychology, 95(3), 274-281. https://doi. org/10.1037/0021-843X.95.3.274

Pennebaker, J., Mehl, M. R., \& Niederhoffer, K. G. (2003). Psychological aspects of natural language use: our words, our selves. Annual Review of Psychology, 54, 47-77. https://doi.org/10.1146/ annurev.psych.54.101601.145041

Pennebaker, J. W., \& Seagal, J. D. (1999). Forming a story: the health benefits of narrative. Journal of Clinical Psychology, 55(10), 1243-1254. https://doi.org/10.1002/(SICI)1097-4679(199 910)55:10<1243::AID-JCLP6>3.0.CO;2-N

Pennebaker, J. W., Kiecolt-Glaser, J. K., \& Glaser, R. (1988). Disclosure of traumas and immune function: health implications for psychotherapy. Journal of Consulting and Clinical Psychology, 56, 239-245. https://doi.org/10.1037//0022-006X.56.2.239

Peñate, W., Del Pino, T., \& Bethencourt, J. (2010). El efecto diferencial de escribir sobre un mismo trauma o sobre diferentes traumas en el bienestar psicológico y el estado de salud auto-informado. Universitas Psychologica, 9(2) 433-445.

Pulverman, C. S., Lorenz, T. A., \& Meston, C. M. (2015). Linguistic changes in expressive writing predict psychological outcomes in women with history of childhood sexual abuse and adult sexual dysfunction. Psychological Trauma: Theory, Research, Practice, and Policy, 7(1), 50-57. https://doi.org/10.1037/a0036462

Quintero, E., Trujillo, Y., \& Reina, M. (2009). Adaptación y validación de los instrumentos de evaluación de ansiedad y depresión de Zung a la lengua de señas colombiana en formato video gráfico (Tesis de pregrado). Universidad Surcolombiana, Neiva, Colombia.

Ramírez-Esparza, N., Pennebaker, J. W., García, F. A., \& Suriá, R. (2007). La psicología del uso de las palabras: un programa de computadora que analiza textos en español. Revista Mexicana de Psicología, 24, 85-99.

Reinhold, M., Bürkner, P., \& Holling, H. (2018). Effects of expressive writing on depressive symptoms-a meta-analysis. Clinical Psychology: Science and Practice. https://doi.org/10.1111/ cpsp. 12224

Rostami, M., Bahmani, B., Bakhtyari, V., \& Movallali, G. (2014). Depression and deaf adolescents: a review. Iranian Rehabilitation Journal, 12(19), 43-53.

Sarmiento-López, J. C., \& Yáñez-Canal, J. (2019). Discusiones políticas y morales referentes a la justicia y su aporte a la psicología del desarrollo moral. Revista Colombiana de Psicología, 28, 165-179. https://doi.org/10.15446/rcp.v28n1.70636

Scruggs, T. E., \& Mastropieri, M. A. (1998). Summarizing single-subject research: issues and applications. Behavior Modification, 22, 221-242.

Sedeño, T. del P. (2011). Variables personales y contextuales que afectan a la eficacia de la reexperimentación emocional (Tesis doctoral). Universidad de la Laguna, San Cristobal, España.

Sloan, D. M., Marx, B. P., \& Greenberg, E. M. (2011). A test of written emotional disclosure as an intervention for posttraumatic stress disorder. Behaviour Research and Therapy, 49(4), 299304. https://doi.org/10.1016/j.brat.2011.02.001 
Smyth, J. M., Hockemeyer, J. R., \& Tulloch, H. (2008). Expressive writing and post-traumatic stress disorder: effects on trauma symptoms, mood states, and cortisol reactivity. British Journal of Health Psychology, 13, 85-93. https://doi. org/10.1348/135910707X250866

Tackman, A. M., Sbarra, D. A., Carey, A. L., Donnellan, M. B., Horn, A. B., Holtzman, ... \& Mehl, M. R. (2019). Depression, negative emotionality, and self-referential language: a multi-lab, multi-measure, and multi-language-task research synthesis. Journal of Personality and Social Psychology, 116(5), 817-834. https://doi.org/10.1037/pspp0000187
Toro, R. A., Avendaño-Prieto, B. L., \& Vargas, N. (2020). Transdiagnostic model of anxiety and depression according to the relationship with affect, uncertainly intolerance, and anxiety sensitivity. Revista CES Psicología, 13(1), 140-152. https://doi. org/10.21615/cesp.13.1.9

Turner, O., K, Windfuhr, K., \& Kapur, N. (2007). Suicide in deaf populations: a literature review. Annals of General Psychiatry, 6(1), 26. https://doi.org/10.1186/1744-859X-6-26 